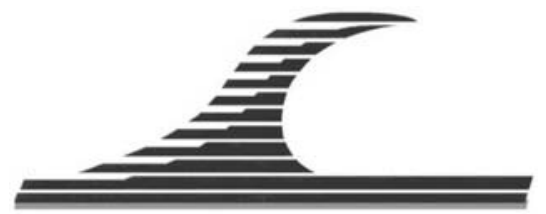

\title{
Elaboration et test d'indicateurs de suivi des initiatives locales de gestion intégrée du littoral méditerranéen
}

\author{
Antoine LAFITTE ${ }^{1}$, Jacques DENIS ${ }^{2}$, Yves HENOCQUE ${ }^{3}$, \\ Bruno ANDRAL ${ }^{4}$, Pierre BOISSERY ${ }^{5}$
}

1. PNUE/PAM/Plan Bleu - Centre d'Activités Régionales, Chargé de mission GIZC, 15 rue Ludwig Van Beethoven, 06560 Valbonne, Sophia Antipolis, France. alafitte@planbleu.org

2. Ifremer, Délégué régional pour les Antilles françaises et Directeur de la station de Martinique, 79 route de pointe Fort, 97231 Le Robert, France.

jacques.denis@ifremer.fr

3. Ifremer, Leader thématique Stratégies Mer et Littoral, Direction Scientifique, 155, rue Jean-Jacques Rousseau, 92138 Issy-les-Moulineaux Cedex, France. yhenocque@ifremer.fr

4. Ifremer, Chef de station, LER-PAC, La Seyne sur Mer, ZIP de Brégaillon, 83500 La Seyne sur Mer, France.bandral@ifremer.fr

5. Agence de l'Eau Rhône, Méditerranée et Corse, Délég. PACA et Corse, Immeuble le Noailles, 62 La Canebière, 13001 Marseille, France.pierre.boissery@eaurmc.fr

\section{Résumé :}

L'évaluation des politiques publiques appliquées à la gestion du littoral relève d'approches relativement récentes. La directive européenne sur l'évaluation stratégique environnementale (ESE) a été transposée en droit français en 2001, au cours de laquelle le législateur a insisté sur la nécessité de disposer d'outils destinés à évaluer des démarches de gestion. Cet article aborde ce sujet en présentant le développement d’une méthode d'évaluation des dynamiques de gestion sur le littoral. Cette méthode prend appui sur les différentes dimensions de l'intégration à laquelle recourt la gestion intégrée des zones côtières. Ainsi, les six indicateurs qui la caractérisent renseignent sur (i) l'approche multi-usage, (ii) la coordination inter-institutionnelle, (iii) l'utilisation à bon escient des connaissances, (iv) l'approche multi-échelle, (v) la participation des acteurs et (vi) la prise en compte de la durabilité. L'approche méthodologique ainsi adoptée a été testée sur cinq sites du littoral méditerranéen français, auprès de cinq groupes d'acteurs représentant (i) les services de l'Etat, (ii) les gestionnaires locaux, (iii) les professionnels du secteur privé, (iv) la société civile et (v) les experts scientifiques.

Soumis le 31 novembre 2011, accepté le 21 septembre 2012, en ligne le 8 octobre 2012.

La seule version examinée est celle écrite en français. La ou les autres versions n'étant pas examinées par le comité de rédaction de la revue, sont donc publiées sous l'entière responsabilité du ou des auteurs.

\section{A TRANSLATED VERSION IN ENGLISH IS AVAILABLE ONLINE}

Pour citer cet article :

LAFITTE A., DENIS J., HENOCQUE Y., ANDRAL B., BOISSERY P. (2012). Elaboration et test d'indicateurs de suivi des initiatives locales de gestion intégrée du littoral méditerranéen. Revue Paralia, Vol. 5, pp 4.1-4.12. 
Au-delà des résultats, cet article met l'accent sur les modalités d'élaboration et de mise en œuvre d'une telle méthode, et discute ses avantages et ses limites d'application en vue d'une possible généralisation à d’autres cas sur le littoral.

\section{Introduction}

Les mesures en faveur de la Gestion Intégrée des Zones Côtières (GIZC) développées par des organisations internationales comme le Programme Mers Régionales du PNUE mais aussi celles exposées par l'approche écosystémique des pêches de la FAO ou encore celles de la Recommandation GIZC de l'Union Européenne, pour ne citer qu'elles, ont toutes souligné l'importance de disposer d'indicateurs d'évaluation de l'impact des activités humaines sur le littoral et de suivi des évolutions de l'état environnemental des milieux marins et côtiers. Le dispositif revient à mesurer l'efficacité des démarches de type GIZC en terme d'amélioration des conditions de gestion de ces espaces. Le guide européen DEDUCE (DEDUCE consortium, 2006) insiste sur le fait que "la littérature et l'expérience pratique ont mis en évidence le besoin de développer des indicateurs pour évaluer la performance (l'exécution) des démarches de GIZC". Selon ce même guide, "il est de plus en plus nécessaire d'évaluer l'efficacité et l'influence des politiques littorales européennes, nationales et locales, particulièrement en matière de développement durable". L'évaluation des modes de gestion sur le littoral ou des politiques publiques en général est donc un élément essentiel, reconnu d'utilité par la majorité des gestionnaires et des décideurs. Malgré tout, il s'agit d'une approche relativement complexe qui doit pouvoir s'adapter au nouveau contexte, de la GIZC et du développement durable en particulier. En effet, "la prise en compte du développement durable nécessite une adaptation des méthodologies d'évaluation des politiques publiques de façon à pouvoir, notamment, "justifier" de leur caractère intégré et concerté" (REY-VALETTE \& ROUSSEL, 2006).

Sur le plan national, les pratiques de gestion intégrée du littoral participent aux politiques territoriales de gestion en accompagnement de la mise en œuvre des outils (LAFITTE, 2008) de gestion proprement dits : SCOT, SAGE, NATURA 2000, Parcs naturels régionaux, les sites du Conservatoire du Littoral, les-contrats de milieux, etc. De telles pratiques font appel aux différentes dimensions de l'intégration qui sont l'objet précisément de la méthode d'évaluation proposée. Ainsi, le but de cette méthode est d'évaluer la manière dont les outils de gestion du littoral sont mis en œuvre en regard des principes et étapes d'un processus de GIZC à l'aide d'un système d'indicateurs déclinant six dimensions de l'intégration. Cette forme d'évaluation des politiques publiques a été conduite auprès des différentes parties prenantes impliquées dans ces processus de gestion par le biais d'enquêtes et d'interviews.

Cette méthode est le fruit d'une étude réalisée dans le cadre de la Convention Cadre établie entre l'Agence de l'Eau Rhône-Méditerranée-Corse et l'Ifremer (Centre de Méditerranée) sur les "Indicateurs de suivi des opérations de gestion intégrée du littoral" 
(LAFITTE, 2010). Cette étude est d’actualité puisque "évaluer les démarches déjà menées en matière de Gestion Intégrée des Zones Côtière" figure en bonne place dans le Livre Bleu des engagements du Grenelle de la mer. En matière de planification spatiale, "donner une dimension véritablement intégrée aux outils de planification territoriale" est également un des engagements du COMOP n6 (GRENELLE DE LA MER, 2010). Les deux principaux axes qui structurent cet article sont (i) la présentation des modalités d'élaboration et de mise en œuvre d'une telle méthode et (ii) la discussion de ses avantages et ses limites d'application.

\section{La méthode de suivi et d'évaluation : une démarche par étape}

La finalité de l'étude est d'évaluer la manière dont les outils de gestion du littoral sont mis en œuvre, en regard des principes et étapes relevant d'un processus de GIZC, pour les traduire plus concrètement en termes d'indice ou de degré GIZC, appliqué à tel ou tel outil de gestion. La mesure de ce degré GIZC recourt à un système d'indicateurs déclinant six dimensions de l’intégration.

La démarche méthodologique se déroule en trois grandes phases qui abordent successivement (i) l'approche qualitative exprimée en terme de prérequis, (ii) l'approche quantitative qui met en jeu les mécanismes de construction de l'information et (iii) l'approche de la représentation synoptique des résultats.

\subsection{Les prérequis de la phase qualitative}

Ils fournissent le matériel méthodologique nécessaire à la mise en place des indicateurs, avant d'entreprendre leur élaboration proprement dite.

\subsubsection{Les sites d'étude}

Les sites d'étude sont au nombre de cinq et appartiennent aux trois régions administratives du Languedoc-Roussillon (un site étudié), de Provence-Alpes-Côte d'Azur (trois sites étudiés) et de la Corse (un site étudié). Ils correspondent à autant d'outils de gestion à analyser.

Le "Contrat de Baie" est singulier dans le sens où cet outil est fondé sur une démarche volontaire et partenariale. Cet outil est intéressant car il s'agit d'un outil de gestion qui, dans les textes, est bien adapté à la gestion du littoral.

Le cas de la "Réserve Naturelle" est intéressant car il vise en priorité la protection du milieu. Avec l'évolution des pressions sur les territoires littoraux, cet outil semble en mesure de s'adapter et de concilier les enjeux de protection avec un développement modéré des activités nautiques.

Le cas des sites "Natura 2000", quant à lui, est également intéressant à étudier car ces périmètres sont à l'origine du qualificatif "plan de gestion" mis en place sur la façade méditerranéenne. Il en est ainsi pour les trois sites étudiés en région PACA : le Cap d’Agde, la rade de Marseille et le Littoral des Maures. La mise en place de ces 
démarches de gestion est issue de modalités de concertation et d'implication des acteurs. Ainsi, le Document d'Objectifs (DOCOB), outil de diagnostic et d'orientation pour la gestion des sites Natura 2000, tente de concilier du mieux possible les impératifs de protection et de développement des espaces sous sa définition.

A souligner que l'historique du site est un aspect important car l'analyse des démarches en cours sera d'autant plus complète qu'elles existent et se déroulent depuis quelques années. Cela permettra donc de bénéficier d'un certain recul. En termes d'ancienneté, les politiques publiques analysées ont en moyenne cinq ans d'existence (sauf pour la Réserve Naturelle de Scandola qui existe depuis trente-cinq ans).

\subsubsection{Les acteurs des outils de gestion}

Ils sont regroupés en cinq catégories et correspondent aux différents collèges représentatifs des parties prenantes de la gestion des territoires retenus. Le choix a été fait d'adapter ces catégories à celles associées aux réflexions dans le cadre du Grenelle de la Mer. A souligner que la représentativité de ces différents acteurs dans le processus de GIZC doit être adaptée selon les spécificités de la zone et de ses enjeux.

La catégorie des "gestionnaires/décideurs" regroupe les élus des collectivités territoriales. Ils apparaissent souvent comme des acteurs centraux. Leur rôle, au-delà de la prise de décisions, tient à leur capacité à fédérer, à promouvoir et à porter un projet à l'échelle territoriale (Région, Département, Commune,...).

Les "représentants de l'Etat" (les préfets, les services déconcentrés de l’Etat,...) et les administrations concernées par la gestion du littoral jouent un rôle majeur pour défendre l’intérêt général et garantir le respect des engagements internationaux et communautaires de la France. Avec la volonté de décentralisation, les rôles de l'état devraient être de plus en plus des rôles d'arbitrage, de soutien technique et de régulation.

Les "acteurs socio-économiques" impliqués traduisent la multiplicité des usages et activités en zone littorale. Ils sont un groupe d'acteurs essentiel dans l'analyse des politiques publiques puisqu'il s'agit de voir de quelle manière ces acteurs locaux sont impliqués dans le processus de gestion et la façon dont ils perçoivent l'action du gestionnaire.

Les "experts techniques et scientifiques" (universitaires, ingénieurs d’étude spécialisés, chercheurs,...) dont les travaux doivent produire des connaissances et des données spécifiques qui alimentent le processus de GIZC. Ces experts éclairent de leurs avis les pouvoirs publics et aident à la prise de décision.

La catégorie de "la société civile" regroupe les citoyens. Ils peuvent être représentés au travers de différentes associations d'usagers ou de protection de l'environnement par exemple. Ce groupe d'acteurs est indispensable à tout processus de gestion intégrée du littoral. 
Pour chaque politique publique analysée, les cinq collèges identifiés ont été auditionnés. En effet, il est indispensable d'avoir une représentativité la plus homogène possible des acteurs pour bénéficier d'une vision la plus objective possible. En tout, 40 personnes ont été ainsi auditionnées, soit en moyenne 8 par site, avec une majorité en faveur des catégories des gestionnaires et des professionnels.

\subsubsection{Les indicateurs et les critères d'évaluation}

Au nombre de six, ces indicateurs ont pour but de mesurer les réels effets de mise en œuvre d'une politique publique au regard des principes de la GIZC. Ces principes sont exprimés selon six déclinaisons de l'intégration qui constitue le "fil rouge" de la présente analyse. Il s'agit de (i) l'intégration horizontale renseignant sur le niveau de prise en compte des objectifs propres à chaque dispositif de gestion considéré, (ii) l'intégration verticale s'intéressant au degré de coordination entre les institutions concernées par la mise en œuvre de ce dispositif, (iii) l'intégration des connaissances exposant le niveau d'intervention des connaissances dans les processus de décision, (iv) l'intégration spatiale évaluant la pertinence des périmètres de gestion, (v) l'intégration participative déterminant le niveau de participation des différents acteurs impliqués dans la gestion et (vi) l'intégration temporelle montrant la projection des actions de gestion dans le temps. Même si une démarche (GOUDEDRANCHE \& GAIGNON, 2006) a été proposée pour l'évaluation des projets GIZC de la DIACT / SG Mer de 2005 "Pour un développement équilibré des territoires littoraux par une GIZC", il n'existe pas à ce jour de consensus ou de méthode standardisée pour construire un système d'indicateurs de GIZC.

A chacun des indicateurs correspondent des critères d'évaluation sous forme de questions posées lors d'interviews au représentant de chacun des cinq collèges en lien avec la dimension d'intégration concernée. Ces critères d'évaluation sont organisés dans un tableau selon un ordre permettant un déroulement optimal des questions durant l'entretien. Ils sont issus de nos réflexions personnelles et d'un travail mené par la Commission Environnement Littoral (CEL, 2002).

\subsection{La construction de l'information de la phase quantitative}

\subsubsection{Système de notation détaillé}

Il s'agit dans cette seconde phase, de procéder à la construction de l'information de chaque indicateur. Ce travail prospectif vise à collecter des informations qui ne sont pas de nature statistique mais plutôt de nature qualitative se rapportant à une situation donnée. Il est néanmoins possible de traiter mathématiquement ce type d'indicateurs. Selon un guide méthodologique (CONSEIL DE L'EUROPE, 2005) "un indicateur peut toujours s'exprimer par un chiffre, qu'il soit qualitatif ou quantitatif". 
A préciser que dans un premier temps et pour plus de simplicité, le choix a été fait d'attribuer le même poids à tous les indicateurs et à tous les collèges.

La fiabilité a été renforcée en posant des questions objectives qui permettent à la personne de cerner progressivement le problème ainsi qu'en interrogeant un échantillon suffisamment représentatif de la population.

Les informations recueillies à la suite des auditions réalisées pour les cinq collèges ont permis d'attribuer une note à chaque critère qui donne ensuite une note générale pour chaque dimension d'intégration (voir figure 1). Si la réponse s’approche de "l’optimum GIZC" initialement défini (un idéal en matière de gestion du littoral vers lequel tendre), la note maximale lui est attribuée. Plus la réponse s'éloigne de cet optimum plus la note diminue. Le barème est donc le suivant : 0 (pas d'intégration), de 0,5 à 1 (intégration faible à moyenne), de 2 à 3 (intégration satisfaisante à bonne). Chaque critère est noté et leur moyenne ("Total" dans la figure 1) est ensuite ramenée sur 5. Il s’agit d'une valeur arbitraire qui est l'échelle d'appréciation des indicateurs.

La notation d'un indicateur résulte toujours du traitement statistique d'un certain nombre de critères s'y rapportant. Le chiffre le plus immédiat et le plus couramment utilisé est la moyenne. Dans le cas présent, le choix s'est porté sur l'utilisation de moyennes simples des notations. Les calculs de probabilité démontrent que, dans tout ensemble relativement homogène, plus le nombre de variables est grand plus la moyenne obtenue est fiable. L'augmentation du nombre de critères est donc un moyen d'augmenter la fiabilité de l'indicateur. Par conséquent, les critères d'évaluation utilisés pour chaque indicateur s’échelonnent de 8 à 19. Une telle différence s'explique par le fait qu'il n'y avait pas suffisamment de matière pour lister de nouveaux critères et équilibrer ainsi les indicateurs. Il s'agit d'un point particulièrement important pour les indicateurs qualitatifs qui ont été élaborés.

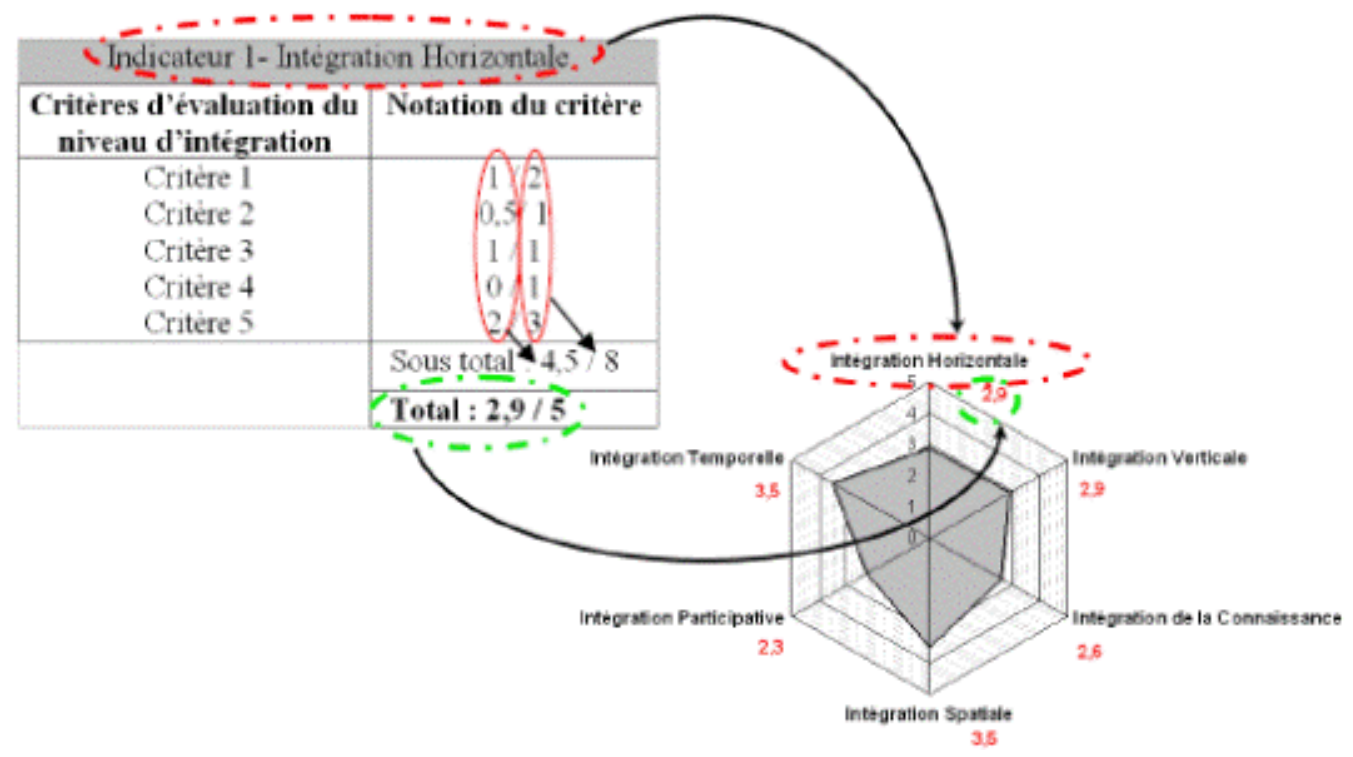

Figure 1. Illustration du système de notation. 


\subsubsection{Elaboration des enquêtes pour renseigner les indicateurs}

Le choix de procéder par enquête auprès des différents groupes d'acteurs est apparu comme la solution la mieux adaptée à notre démarche, par rapport aux types de données que nous recherchions. Le questionnaire et l'enquête sont deux éléments essentiels de la démarche adoptée. Le questionnaire est de type "oral" et l'enquête est de type "face à face" ou "enquête par entretien personnel" (PNUE/PAM/SMAP III PROJET, 2006). L'enquête reste la méthode de prédilection pour collecter des données car elle est nettement plus efficace pour améliorer la qualité des informations recueillies. Elle permet en effet de rendre l'échange interactif, de relancer l'auditeur ou de le recentrer sur le sujet principal. Les taux de réponse sont habituellement bien supérieurs à ceux obtenus lors des autres types d'enquêtes (par téléphone, courriel ou courrier). La façon de procéder comporte deux étapes. La première consiste à cibler les personnes les plus représentatives des démarches de gestion et à fixer un rendez-vous. La seconde étape constitue l'audition au moyen de l'enquête. Le fait de rencontrer des représentants de différents collèges lors d'auditions a permis de renseigner de façon quasiment complète les critères qualificatifs des indicateurs. Ainsi, pour chaque site analysé, un recueil des propos des personnes auditionnées a été constitué pour justifier et nuancer au maximum les notes attribuées. A noter que les questionnaires, les indicateurs et les critères n'ont pas été revus après chaque audition, l'idée étant de disposer d'une même base de questionnaire pour l'analyse. L'enquête durait en moyenne 1h30 (de 45 minutes pour la plus rapide à $3 \mathrm{~h}$ pour la plus longue). Le taux de réponses moyen à l'issue des 40 entretiens est de $90 \%$.

\subsubsection{Traitement des enquêtes}

Le premier niveau concerne les observations de l'enquêté. Lors de chaque entretien, toutes les remarques ou commentaires formulés par la personne auditionnée ont été enregistrées. Des observations portant sur l'utilité de l'étude, sur la méthode employée et sur les indicateurs ont été énoncées. Il a donc été possible de rédiger un point de vue relatif à la façon dont les enquêtés ont perçu l'entretien tant sur la forme que sur le fond. Il s’agit d'un précieux retour d'expérience. Le second niveau expose le ressenti de l'enquêteur après chaque entretien à propos de l'audition, de la fonction (rôle et place) de l'enquêté dans le dispositif et de son comportement (en lien avec sa personnalité).

\subsection{La visualisation de l'information par la représentation synoptique}

2.3.1 Un radar à 6 axes (un par indicateur) matérialise schématiquement l'ensemble des valeurs obtenues par collège pour un site donné

A chacune des valeurs est attachée sa fourchette de variation avec les valeurs minimales et maximales. La fiche de reportage synthétise les résultats obtenus (voir figure 2 et tableau 1) pour les six indicateurs correspondant à chaque politique publique analysée. 


\subsubsection{Une carte de situation des sites étudiés : un état de référence en 2010}

Le produit final de restitution est une carte panoramique à l'échelle de la façade méditerranéenne (voir figure 3). Elle permet de visionner le positionnement spatial des politiques de gestion analysées et leurs caractéristiques grâce à un tableau croisé "indicateurs/groupe d'acteurs". Les résultats sont représentés par un code couleur attribué à chaque indicateur sur l'échelle de 0 à 5 des "radars", pour distinguer les niveaux faible (valeurs de 0 à 3), modéré (de 3.1 à 4), satisfaisant (de 4.1 à 4.5) et excellent (de 4.6 à 5).

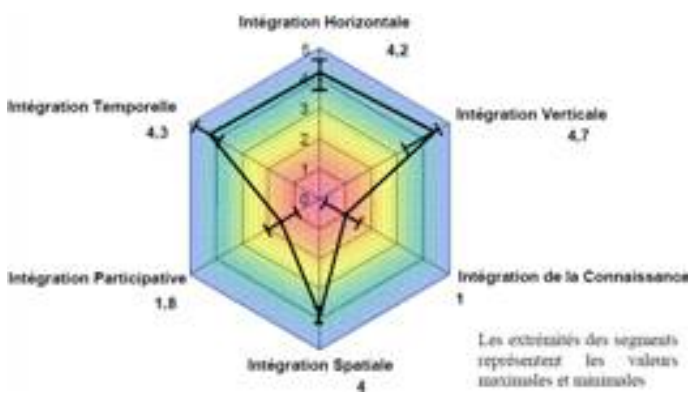

Figure 2. Exemple hypothétique de représentation de l'information en "radar" selon les cinq collèges et les six indicateurs.

\begin{tabular}{|c|c|c|c|c|c|c|}
\hline $\begin{array}{l}\text { Nom de la politique publique } \\
\text { analysie }\end{array}$ & $\begin{array}{l}\text { Services de } \\
\text { réat }\end{array}$ & $\begin{array}{l}\text { Gestionanares locoux } \\
\text { (Gestionnare } \\
\text { Colletivité locale) }\end{array}$ & Professionnels & $\begin{array}{l}\text { Socitite } \\
\text { Civile }\end{array}$ & \begin{tabular}{|c|} 
Experts \\
Scientifiques
\end{tabular} & \\
\hline \begin{tabular}{|l|} 
Intégration Horizontale \\
\end{tabular} & 4 & 4,8 & 4,2 & 4,2 & 4 & $\begin{array}{l}4,6 \text { a } 5 \text { : Excellent } \\
4,1 \text { a } 4,5: \text { : }\end{array}$ \\
\hline Intégration Verticale & 4,3 & 4,8 & 4,1 & 4,8 & 4,1 & 3,1 a d 4 : Modéré \\
\hline Integration des Connaissances & 1,2 & 2,1 & 2 & 1,4 & 1 & 1' à 3 : Faible \\
\hline Intégration Spatiale & 4 & 4,1 & 4,8 & 4,1 & 4,8 & \\
\hline Intégration Participative & 2,1 & 2,5 & 1,3 & 1,1 & 1,2 & \\
\hline Intégration Temporelle & 4 & 4,1 & 4,2 & 4,8 & 4,6 & \\
\hline
\end{tabular}

Tableau 1. Les différentes perceptions hypothétiques des représentants des cinq collèges pour chacun des six indicateurs.

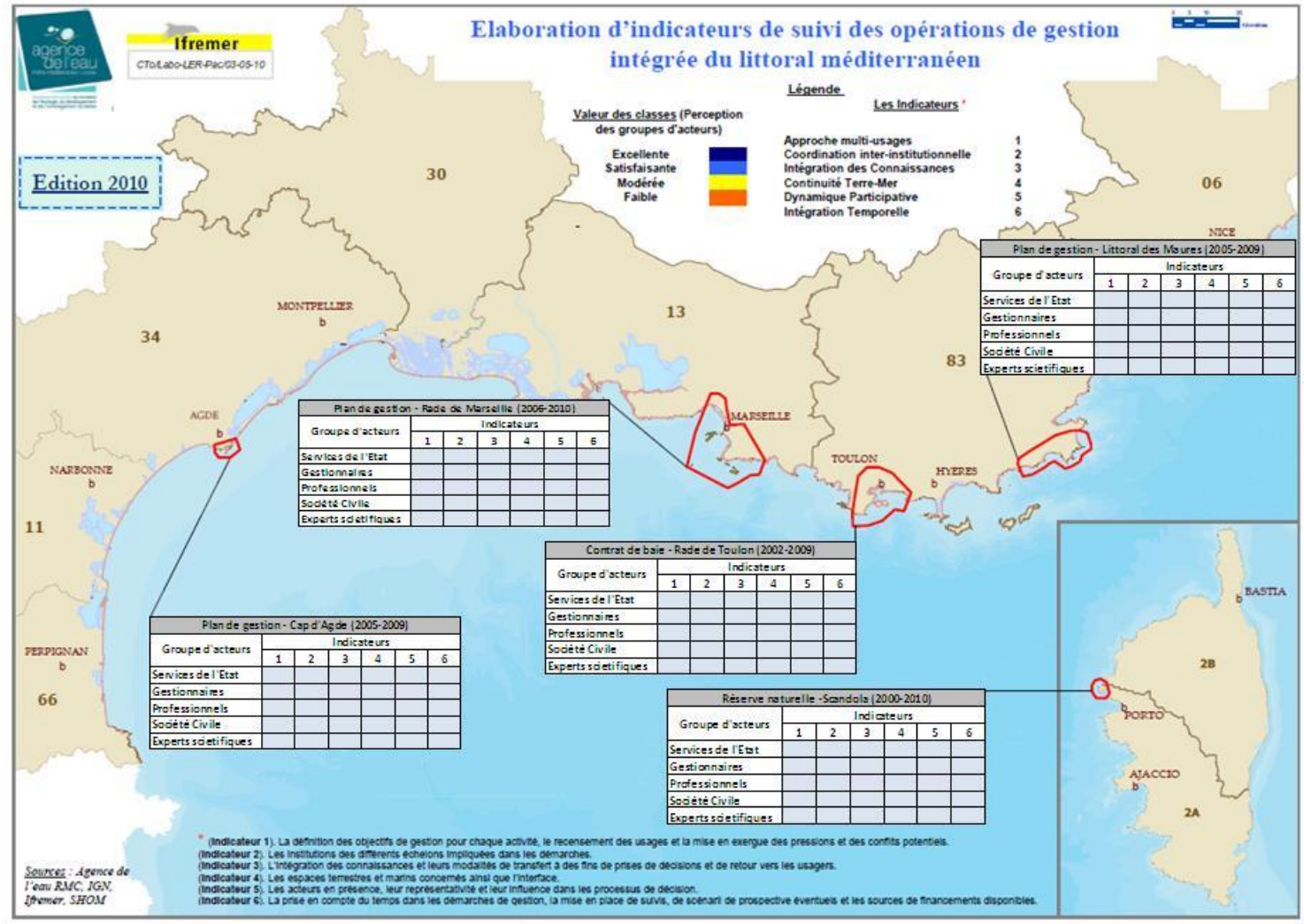

Figure 3. Tableau de bord des résultats pour chaque politique publique analysée. 


\section{Discussion}

\subsection{Les atouts d'une telle approche}

Cette évaluation a permis de mettre en valeur les points forts de ces pratiques de gestion mais aussi les facteurs qui limitent l'approche intégrée au sein des politiques publiques. L'intérêt de cette étude est donc d'apporter un retour aux gestionnaires, en s'appuyant sur la perception d'un certain nombre d'acteurs locaux pour savoir de quelle manière les actions de gestion engagées sur un territoire sont perçues par ces derniers. L'idée étant, à terme, de donner un éclairage aux gestionnaires sur les points faibles de façon à les améliorer et sur les points forts à conserver ou à consolider en vue d'aboutir à une gestion résolument intégrée des espaces concernés.

Grâce aux six indicateurs définis et aux différents critères d'évaluation du niveau de GIZC, une première évaluation d'outils de gestion a été possible. Cette démarche est apparue, pour la majorité des personnes rencontrées, intéressante et bien nécessaire. Selon leurs dires, évaluer la pertinence d'un mode de gestion est utile car il est important pour le gestionnaire de se poser la question suivante : "qu'est ce qui a été fait et que reste-t-il à faire ?".

Au travers de cette étude, il a été question d'étudier l'application locale d'un outil de gestion. Grâce à cette méthode, une première base de travail se dessine pour analyser la mise en œuvre, la manière dont un outil de gestion est mis en place sur un territoire. La plupart des personnes auditionnées ont ainsi mentionné l'aspect suivant : "L'entretien permet à l'enquêté de se plonger dans la politique publique à laquelle il est confronté quotidiennement. Votre étude permet à toutes les personnes auditionnées de formuler leur opinion à un moment donné".

A souligner que cette démarche peut être applicable et reproductible sur différents sites.

\subsection{Les limites de la démarche}

\subsubsection{Sur un plan formel (le rendu et les collèges auditionnés)}

Le choix de représentation schématique et synthétique finale sous forme de "radar" à six côtés présente le risque que les lecteurs ne retiennent que cette conclusion illustrée. Cette représentation est néanmoins utile pour illustrer et suggérer de façon synoptique "le niveau de GIZC" d'un outil. Certains gestionnaires ont ainsi précisé : "la vision en radar qui sortira au final est intéressante car nous pourrons nous imager la façon dont nos actions sont perçues". Cependant, cette représentation n’est pas forcément la plus pertinente. En effet, bien que les extrêmes soient représentés graphiquement par des segments, elle montre aussi une agrégation des résultats sous la forme d'une moyenne. Il ne faut donc pas se limiter à cette simple représentation. C'est pour cela que les perceptions et les remarques des différents groupes d'acteurs auditionnés sont 
essentielles pour donner plus de discernement et de matière pour la compréhension de ces "radars".

Concernant les groupes d'acteurs, sur l'ensemble des cinq sites étudiés, les perceptions variaient selon les collèges. Ainsi, le collège "Etat" est le plus satisfait des modes de gestion mis en place. Viennent ensuite les "gestionnaires" et les "experts scientifiques". Les collèges "professionnels" et "Société Civile" sont en majorité plus réservés sur l'aspect intégré des outils de gestion considérés. Pour mener une analyse relativement complète et équilibrée, il n'est donc pas possible de mettre de côté un collège au risque de biaiser fortement les résultats puisque chaque groupe d'acteurs dispose de sa propre vision.

La fonction occupée par la personne auditionnée est essentielle à prendre en considération. En effet, si nous prenons l'exemple du collège "Société Civile", la vision diffère si nous auditionnons une association de protection de la nature, de plaisanciers ou encore de randonneurs. Le même constat peut être souligné chez les professionnels. En effet, leurs attentes et leurs points de vue diffèrent s'il s'agit d'un pêcheur, d'un plongeur, d'un exploitant de vedettes à passagers ou d'un directeur de chantier naval. Cet aspect n’est pas directement pris en compte dans la méthode proposée.

L'attitude des représentants est également un aspect important à considérer. Le fait que la personne auditionnée soit favorable ou non à la politique de gestion menée entraînera inévitablement des différences de réponses qui joueront dans la notation. Ainsi, pour une même question, sur un même objet, nous aurons deux réponses distinctes. L'évaluation de la mise en œuvre d'un outil de gestion est donc fortement liée à la perception de chaque individu.

\subsubsection{Concernant le fond (les indicateurs et les critères)}

Bien qu'ayant essayé de varier les sources, en auditionnant une personne pour chacun des cinq collèges, le fait de ne cibler qu'une personne par collège n'est peut-être pas suffisant pour obtenir des statistiques exploitables. Une personne par collège est a priori suffisante dans le sens où cette personne a été ciblée comme étant la plus représentative de son collège pour le site analysé. Par exemple, pour le collège des "experts scientifiques", le président du comité scientifique, coordinateur d’une équipe pluridisciplinaire, a été auditionné. De plus, cet aspect est compensé par l'audition de plus d'une personne par collège (nombre initialement prévu au départ). A rappeler aussi que le but de cette étude a une portée essentiellement méthodologique.

Deux éléments sont également ressortis concernant la perception des gestionnaires sur la méthode proposée. Le premier concerne l'exercice difficile que demande la synthèse d'un certain nombre d'années d'expérience en un laps de temps relativement court (celui de l'entretien). Le second point est que la démarche leur semble assez théorique. "Nous devons rentrer dans des cases alors que la réponse que l'on donne doit toujours être nuancée". 
Concernant les critères, ils ne sont pas identiques en nombre entre chacun des six indicateurs. Cela se traduit par un manque de fiabilité et d'égalité entre ces derniers en termes de notation. En effet, un critère discriminant aura une répercussion plus importante sur la somme de tous les critères lorsque ces derniers sont peu nombreux dans un indicateur. Autrement dit, dans l'indicateur 1 (intégration horizontale), composé de 8 critères, si un des critères obtient la note "0", celle-ci aura plus d'effet sur la moyenne que dans l'indicateur 3 (intégration des connaissances), composé lui, de 19 critères. Une autre remarque concerne l'ambigüité sensible de certains critères. La nuance entre deux questions n'est pas toujours flagrante. C'est pour cela que l'entretien en face à face est en partie indispensable. Cela permet d'expliquer à notre auditeur ce que nous voulons cibler au travers de la question posée.

Par ailleurs, il a été constaté au cours des auditions que certains critères sont plus orientés vers les gestionnaires. Ainsi, par manque de savoirs, les autres collèges ne sont pas en mesure d'apporter une réponse à la question posée.

Enfin, malgré le travail bibliographique et de construction des indicateurs, il se peut que certains éléments importants pour l'analyse du degré de GIZC aient été omis. Cette question a donc été posée à chaque enquêté. Ainsi, à la question "Selon vous, un ou plusieurs aspects indispensables à ce type d'évaluation auraient-ils été omis ? ", seuls 5 enquêtés sur 40 ont répondu "oui" et ont apporté leur point de vue.

Malgré ces remarques, la tentative d'être le plus exhaustif possible en couvrant la plupart des champs avec les 84 critères a permis de mener une première démarche d'évaluation des politiques publiques en matière de GIZC. Pour illustrer cet aspect, certains enquêtés ont tenu les propos suivants : "les indicateurs couvrent plutôt bien les questions relatives aux problématiques de gestion et les questions (critères) que vous posez sont pertinentes".

\section{Conclusion et recommandations}

Il parait délicat de proposer une fréquence d'analyse identique pour tous les sites dans la mesure où chacune d'eux a son propre déroulement. Ce qui est valable pour toutes les politiques c'est qu'une analyse annuelle parait assez peu pertinente. En effet, l'analyse de l'évolution des pratiques et des façons dont les gestionnaires gèrent l'espace sont difficilement mesurables sur le court terme. En revanche, mener une analyse pour comparer deux situations sur un même site à une échelle de révision du plan de gestion parait plus rigoureux et significatif en termes de résultats avancés.

L’échantillonnage à cibler pour les enquêtes est un élément essentiel car la perception sur les modes de gestion en place des personnes auditionnées fournit les résultats. Etant donné que l'analyse porte sur des modes de gestion locaux, il parait plus pertinent d'insister sur les perceptions des gestionnaires et des professionnels. Il est alors proposé d'auditionner au minimum un représentant pour chacune des activités professionnelles de l'espace côtier considéré et un ou plusieurs gestionnaires, ce nombre étant fonction 
de la structure porteuse. Les perceptions des autres collèges, à savoir les "experts scientifiques", la "société civile" et les "services de l'Etat" sont également à prendre en compte. Le nombre de leurs représentants peut-être plus faible (un ou deux).

La représentation des résultats "en radar" est un aspect déjà traité dans les limites de la méthodologie mais il est recommandé d'associer systématiquement à cette représentation le tableau des résultats et le celui avec les codes couleurs présentés sur le tableau de bord.

\section{Références bibliographiques}

CEL -Commission Environnement Littoral- (2002). Pour une approche intégrée de gestion des Zones Côtières. Rapport au Gouvernement, 82 p.

CONSEIL DE L'EUROPE (2005). Elaboration concertée des indicateurs de la cohésion sociale. Guide méthodologique, 236 p.

DEDUCE consortium (2006). Mettre en œuvre une approche basée sur les indicateurs pour évaluer le développement durable sur le littoral. Guide d'utilisation des indicateurs, 70 p. [URL http://www.deduce.eu/results.html].

GOUDEDRANCHE L., GAIGNON J.L. (2006). Suivi - Accompagnement des projets de GIZC, résultats de la première enquête. $6^{\text {ièmes }}$ Journées Scientifiques et Techniques du CETMEF, Paris, 4p.

GRENELLE DE LA MER (2010). Comité Opérationnel $n^{\circ} 6$ : Aménagement, Protection et Gestion des espaces littoraux. Rapport d'étape, 111 p.

LAFITTE A. (2008). Les outils de la GIZC. Mémoire de Master 2 option: Développement Durable, Conflits d'usage et GIZC, Université de Nantes, 192 p.

LAFITTE A. (2010). Elaboration d'indicateurs de suivi des opérations de gestion intégrée du littoral méditerranéen : approche méthodologique appliquée à cinq sites d'étude. Direction des Opérations / Laboratoire Environnement Ressources Provence Azur Corse (DOP/LER-PAC). Convention n²009 / 095. Accord cadre Agence de l’Eau RM\&C / Ifremer. 77 p.

PNUE/PAM/SMAP III PROJET (2006). Sensibilisation et création d'un cadre politique pour l'intégration de l'environnement et du développement en Méditerranée. Manuel pour la réalisation du sondage d'opinion, un guide pour la préparation et la mise en œuvre de l'enquête sur la prise de conscience de l'état de l'environnement, Split, 24 p.

REY-VALETTE H., ROUSSEL S. (2006). L'évaluation des dimensions territoriale et institutionnelle du développement durable : le cas des politiques de Gestion Intégrée des Zones Côtières. Revue Développement durable et territoire, dossier 8 : "Méthodologies et pratiques territoriales de l'évaluation en matière de développement durable", mis en ligne le 13 décembre 2006, 22 p. [URL : http://developpementdurable.revues.org/document3311.html]. 